Wilkesmann, Uwe

Wettbewerb und Hierarchie versus Markt- und Wahrheit-Fetisch Allokationsmechanismen in der (Erziehungs-)Wissenschaft

Erziehungswissenschaft 27 (2016) 53, S. 43-51

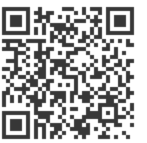

Quellenangabe/ Reference:

Wilkesmann, Uwe: Wettbewerb und Hierarchie versus Markt- und Wahrheit-Fetisch -

Allokationsmechanismen in der (Erziehungs-)Wissenschaft - In: Erziehungswissenschaft 27 (2016) 53, S. 43-51 - URN: urn:nbn:de:0111-pedocs-127512 - DOI: 10.25656/01:12751

https://nbn-resolving.org/urn:nbn:de:0111-pedocs-127512

https://doi.org/10.25656/01:12751

in Kooperation mit / in cooperation with:

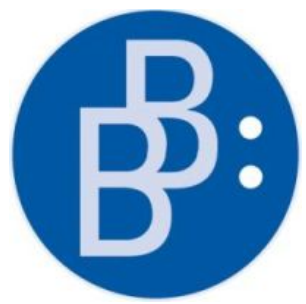

https://www.budrich.de

\title{
Nutzungsbedingungen
}

Gewährt wird ein nicht exklusives, nicht übertragbares, persönliches und beschränktes Recht auf Nutzung dieses Dokuments. Dieses Dokument ist ausschließlich für den persönlichen, nicht-kommerziellen Gebrauch bestimmt. Die Nutzung stellt keine Übertragung des Eigentumsrechts an diesem Dokument dar und gilt vorbehaltlich der folgenden Einschränkungen Auf sämtlichen Kopien dieses Dokuments müssen alle Urheberrechtshinweise und sonstigen Hinweise auf gesetzlichen Schutz beibehalten werden. Sie dürfen dieses Dokument nicht in irgendeiner Weise abändern, noch dürfen Sie dieses Dokument für öffentliche oder kommerzielle Zwecke vervielfältigen, öffentlich ausstellen, aufführen, vertreiben oder anderweitig nutzen.

Mit der Verwendung dieses Dokuments erkennen Sie die Nutzungsbedingungen an.

$$
\text { Dokuments erkennen Sie die }
$$

\section{Terms of use}

We grant a non-exclusive, non-transferable, individual and limited right to using this document.

This document is solely intended for your personal, non-commercial use. Use of this document does not include any transfer of property rights and it is conditional to the following limitations: All of the copies of this documents must retain all copyright information and other information regarding legal protection. You are not allowed to alter this document in any way, to copy it for public or commercial purposes, to exhibit the document in public, to perform, distribute or otherwise use the document in public.

By using this particular document, you accept the above-stated conditions of use.

\section{Kontakt / Contact:}

\section{peDOCS}

DIPF | Leibniz-Institut für Bildungsforschung und Bildungsinformation Informationszentrum (IZ) Bildung

E-Mail: pedocs@dipf.de

Internet: www.pedocs.de

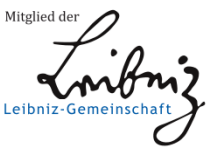




\section{INHALTSVERZEICHNIS}

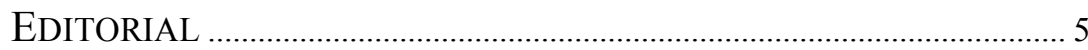

BEITRÄGE ZUM THEMA

„HAMSTERRAD (ERZIEHUNGS-)WISSENSCHAFT: PREKÄR UND BESCHLEUNIGT?“‘

Carsten Bünger, Kerstin Jergus \& Sabrina Schenk

Prekäre Pädagogisierung: Zur paradoxen Positionierung des erziehungswissenschaftlichen „Nachwuchses“.

Andrea Lange-Vester

Zwischen W3 und Hartz IV: Zumutungen prekarisierter

Arbeitsbedingungen für den (erziehungs-)wissenschaftlichen

Nachwuchs

Sigrid Metz-Göckel

Prekarität, Geschlechterkonstellationen und Elternschaft im

wissenschaftlichen Mittelbau

Uwe Wilkesmann

Wettbewerb und Hierarchie versus Markt- und Wahrheit-Fetisch -

Allokationsmechanismen in der (Erziehungs-)Wissenschaft

Anna Schütz, Nina Blasse \& Doris Wittek

Die Suche nach einer neuen Normalität: Disziplinpolitische

Perspektiven auf die Prekarität im erziehungswissenschaftlichen Feld

ALLGEMEINE BEITRÄGE

Gerd Hölter

Bildungsforschung im Konflikt von administrativer Über- und reflektiver Untersteuerung: Ein Erfahrungsbericht aus einem BMBF-Forschungsprojekt zur frühen Kindheit 


\section{MitTEILUNGEN DES VORSTANDS}

Protokoll der ordentlichen Mitgliederversammlung der Deutschen

Gesellschaft für Erziehungswissenschaft e.V.

Anlage zum Protokoll der ordentlichen Mitgliederversammlung der

Deutschen Gesellschaft für Erziehungswissenschaft e.V.

Satzung der Deutschen Gesellschaft für Erziehungswissenschaft e.V ............. 81

Ethik-Kodex der Deutschen Gesellschaft für Erziehungswissenschaft............ 89

Call for Papers zur Fachtagung der Deutschen Gesellschaft für

Erziehungswissenschaft

\section{BERICHTE AUS DEN SEKTIONEN}

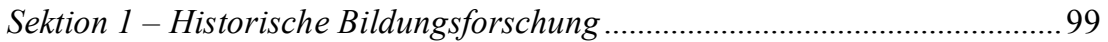

Sektion 2 - Allgemeine Erziehungswissenschaft ............................................ 102

Sektion 3 - Interkulturelle und International Vergleichende Erziehungswissenschaft (SIIVE) ................................................. 106

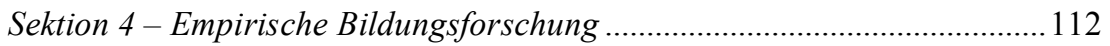

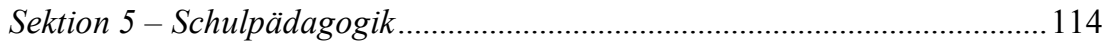

Sektion 8 - Sozialpädagogik und Pädagogik der frühen Kindheit.................. 118

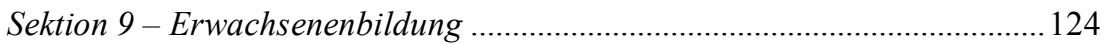

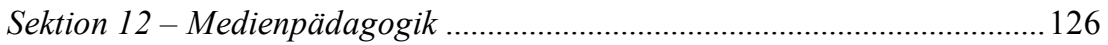

NOTIZEN

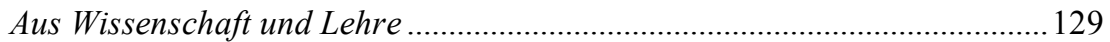

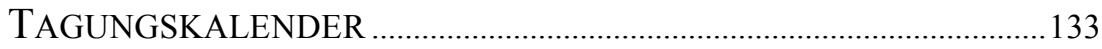

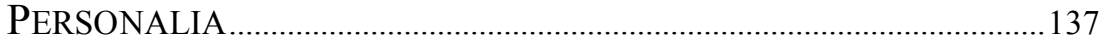

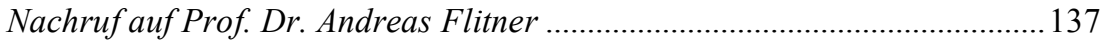

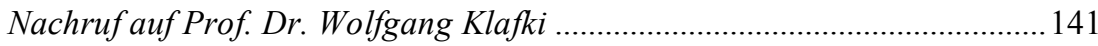




\section{EDITORIAL}

Die Datenlage ist eindeutig: Der Bundesbericht Wissenschaftlicher Nachwuchs weist die Dynamik der Prekarisierung von Beschäftigungsverhältnissen im akademischen Feld jenseits der Professuren für die zweite Hälfte der 2000er-Jahre mit den folgenden Worten aus: „Der Anteil an befristet beschäftigten wissenschaftlichen Mitarbeiterinnen und Mitarbeiter stieg seit 2005 von 79 auf 90\%" (Konsortium Bundesbericht Wissenschaftlicher Nachwuchs 2013, S. 185).

Differenziert man diese Konstellation nochmals zwischen Teilzeit- und Vollzeitbeschäftigten, wird deutlich, dass die Figur des Nicht-Prekären, also des unbefristet Beschäftigten, überhaupt nur in einer merklichen Größe unter den Vollzeitbeschäftigten (14 Prozent) für das Arbeitsfeld bundesdeutscher Hochschulen nachweisbar ist. Unter den teilzeitbeschäftigten Mittelbau-Angehörigen finden sich dagegen nur noch fünf Prozent unbefristet beschäftigte Wissenschaftlerinnen und Wissenschaftler. Zugleich diagnostizieren zeitsoziologische Analysen insbesondere für die Arbeitswelt in den Gegenwartsgesellschaften eine Beschleunigungstendenz. Im akademischen Arbeitsfeld werden u.a. die Unwucht in der Studierenden-Lehrenden-Relation, die Managerialisierung und die Kommerzialisierung der Hochschuladministration und die Standardisierung der Studienorganisation für einen stärker verdichteten Arbeitsalltag verantwortlich gemacht. Vielfach dominiert daher der Eindruck unter Wissenschaftlerinnen und Wissenschaftlern, mit der beruflichen Entscheidung für das akademische Feld sei man in ein „Hamsterrad der Wissenschaft" eingetreten. Gehen also in diesem „Hamsterrad (Erziehungs-) Wissenschaft" Prekarisierung und Beschleunigung eine Allianz ein? Diese Frage steht im Fokus des thematischen Schwerpunkts der vorliegenden 53. Ausgabe der Erziehungswissenschaft. Ihrer Beantwortung widmen sich die folgenden Beiträge:

Carsten Bünger, Kerstin Jergus und Sabrina Schenk eröffnen mit ihrem Beitrag die Diskussion zum Schwerpunkt. Sie loten die Möglichkeiten einer erziehungswissenschaftlichen Reflexion der zunehmenden Prekarisierung wissenschaftlicher Beschäftigungsverhältnisse jenseits der Lebenszeitprofessur aus. Eine entsprechende Analyse habe ihres Erachtens, erstens den spezifischen Modus der Subjektivierung „angehender“ Wissenschaftlerinnen und Wissenschaftler zu rekonstruieren. Sie solle zweitens die Tatsache der gesellschaftspolitischen Blindstelle erziehungswissenschaftlicher Debatten zur Kenntnis nehmen, was eine systematische Ausblendung der Bedingungen wissenschaftlicher Produktion zur Folge habe. Und drittens habe sie den pädagogisierten Verdeckungszusammenhang in der universitären Anerken- 
nungsstruktur aufzuschließen, der Hierarchien in Betreuungs- und Bewährungsverhältnisse umformatiere.

Ausgehend von der Diagnose einer zunehmenden Prekarisierung, die auch für das erziehungswissenschaftliche Feld nachzuzeichnen sei, skizziert Andrea Lange-Vester den gegenwärtigen Kontext des akademischen Arbeitsfeldes. In diesen stellt sie Forschungsbefunde aus einer eigenen Studie zur Etablierungspraxis junger Wissenschaftlerinnen und Wissenschaftler. Damit weist Andrea Lange-Vester auf Aspekte hin, mit denen sich die befristet Beschäftigten im akademischen Feld auseinandersetzen müssen und die Einfluss auf ihre Etablierung in der (Erziehungs-)Wissenschaft haben: Die Familienplanung der Wissenschaftlerinnen und Wissenschaftler, ihre räumliche Mobilität sowie ihre Bereitschaft, trotz prekärer Arbeitsbedingungen ein hohes Engagement als notwendige Anforderung anzusehen, sind zentrale Aspekte, auf die sie damit aufmerksam macht. Darüber hinaus kann Lange-Vester zeigen, wie milieu- und herkunftsspezifische Dimensionen wirken: Bildungsaufsteigende weisen immer wieder eine geringere Passung zu den Herausforderungen des akademischen Feldes auf und werden deshalb leichter vom Aufstiegsweg verdrängt.

Sigrid Metz-Göckel diskutiert in ihrem Beitrag die widersprüchliche Einheit von wissenschaftlicher und sorgender Arbeit. Dabei hebt sie u.a. den weit verbreiteten unerfüllten Kinderwunsch von Wissenschaftlerinnen und Wissenschaftlern hervor. Sie weist außerdem auf eine unerwartet geringere Erschöpfungssymptomatik unter Wissenschaftlerinnen mit Kind(ern) hin und zeigt zugleich auf, dass Wissenschaftlerinnen mit Kind immer wieder aufgrund fehlender Vereinbarkeit von Beruf und Familie aus dem akademischen Feld aussteigen - trotz des im Vergleich zu anderen Arbeitsfeldern relativ hohen Anteils an Wissenschaftlerinnen in der Erziehungswissenschaft. Im Widerspruch zu der vielfachen, häufig zertifizierten Familienorientierung bundesdeutscher Universitäten ist daher mit Metz-Göckel von einer „strukturelle(n) Rücksichtslosigkeit“ dieser Organisation gegenüber Eltern auszugehen.

Die Frage nach der Akzeptanz bestehender Allokationsmechanismen unter Rektoren/Präsidien und Professorinnen sowie Professoren vor dem Hintergrund der veränderten Steuerungslogiken an bundesdeutschen Hochschulen stellt Uwe Wilkesmann ins Zentrum seiner Überlegungen. Er fragt dabei nach einer möglichen Veränderung dieser Akzeptanz von Allokation auf Seiten der in der Hochschulhierarchie entscheidenden Akteure. Für das akademische Feld identifiziert Uwe Wilkesmann einen ,inszenierten Wettbewerb“, also eine spezifische Marktlogik, die sich von denen in anderen gesellschaftlichen Subsystemen unterscheide. Empirisch unterscheidet Wilkesmann drei Reaktionstypen in der neu gesteuerten Hochschule (New Public Management) und markiert schlussfolgernd die Relevanz hierarchischer Entscheidungen, die sich nicht unter einem „Markt-Fetisch“ subsumieren lassen. 
Anna Schütz, Nina Blasse und Doris Wittek diskutieren in ihrem Beitrag disziplinpolitische Perspektiven auf Prekarität im Feld der Erziehungswissenschaft. Ein Stabilisierungspotenzial für die gerade auch im internationalen Vergleich massiv ausgeprägte Prekarität im akademischen Feld sehen Schütz, Blasse und Wittek darin, dass diese von vielen Wissenschaftlerinnen und Wissenschaftlern nicht nur in Kauf genommen, sondern darüber hinaus nicht hinterfragt werde. Gründe dafür sehen die Autorinnen darin, dass das wissenschaftliche Berufsfeld der sachbezogenen intrinsischen Motivation junger Wissenschaftlerinnen und Wissenschaftler Raum gebe, ihnen vermeintlich erleichterte Einstiegsmöglichkeiten biete und über den Erwerb universitärer Qualifikationsgrade respektive Titel ein weiterhin hohes gesellschaftliches Prestige in Aussicht stellen könne. Die gegenwärtige Prekarisierungsdynamik verorten die Autorinnen ebenfalls in der veränderten Steuerungs- und Regulierungslogik bundesdeutscher Hochschulen, aber auch in den weiterhin stabilen hierarchischen Abhängigkeitsverhältnissen, denen nicht-professorale Wissenschaftlerinnen und Wissenschaftler unterliegen. In Bezug auf mögliche disziplinpolitische Konsequenzen sehen Schütz, Blasse und Wittek vor allem die Fachgesellschaft sowohl als Ort der selbstkritischen Vergewisserung als auch als (politische) Akteurin in der wissenschaftspolitischen Auseinandersetzung um den Arbeitsmarkt „Wissenschaft“ in der Pflicht.

Unter der Rubrik „Allgemeine Beiträge“ legt Gerd Hölter einen mit dem Schwerpunkt dieser Ausgabe der Erziehungswissenschaft korrespondierenden Erfahrungsbericht aus dem Kontext eines Forschungsprojektes zu Fragen der frühen Kindheit vor. Er konstatiert eine zunehmende Durchsetzung des „ökonomischen Denkens" auch in erziehungswissenschaftlichen Bereichen der frühen Kindheit und weist auf die damit verbundene Gefahr hin, dass Traditionen der Allgemeinbildung und des damit verbundenen kritischen Denkens verloren gehen. Unter Verweis auf eigene Erfahrungen in einem BMBF-Verbund zieht Hölter die Möglichkeit in Betracht, dass Veränderungen innerhalb der Forschungsförderungs- und -steuerungspolitik derartige Entwicklungen stabilisieren können.

Im Anschluss an die inhaltlichen Beträge erfolgen die „Mitteilungen des Vorstands. Hier finden Sie das Protokoll der ordentlichen Mitgliederversammlung mit seiner Anlage, die Satzung und den geänderten Ethikkodex der DGfE. Nach den „Berichten aus den Sektionen“ und den "Notizen“ schließen der „Tagungskalender“ und die Rubrik „Personalia“ diese Ausgabe der Erziehungswissenschaft. 


\title{
Wettbewerb und Hierarchie versus Markt- und Wahrheit-Fetisch - Allokationsmechanismen in der (Erziehungs-)Wissenschaft
}

\author{
Uwe Wilkesmann
}

\section{$1 \quad$ Einleitung}

In der Wissenschaft, wie in allen anderen gesellschaftlichen Subsystemen, muss die Frage der Allokation knapper Güter gelöst werden. Knappe Güter sind dabei Stellen und Geld für Wissenschaftlerinnen und Wissenschaftler sowie Karriereoptionen. Die beiden Forschungsfragen, die in diesem Artikel beantwortet werden sollen, lauten: Welche Allokationsmechanismen existieren für diese knappen Güter sowohl im Wissenschaftssystem als auch in der Organisation Hochschule? Wie nehmen Wissenschaftlerinnen und Wissenschaftler diese Steuerungsinstrumente wahr bzw. welche Einstellung haben sie zu ihnen? Es wird argumentiert, dass das Allokationsprinzip des Wissenschaftssystems ein inszenierter Wettbewerb ist, der im Schatten der Hierarchie steht. In der Organisation Hochschule ist der Allokationsmechanismus die hierarchische Entscheidung. Empirisch wird dann überprüft, ob die Akzeptanz dieser Steuerung bei den Rektorinnen sowie Rektoren und bei den Professorinnen sowie Professoren unterschiedlich ausgeprägt ist und sich möglicherweise über die Zeit verändert hat.

\section{Inszenierter Wettbewerb und hierarchische Entscheidung versus Markt- und Wahrheit-Fetisch}

Generell müssen zwei Ebenen der Allokation unterschieden werden: das Wissenschaftssystem und die Organisation Hochschule. Für letztere stellt erstere die Umwelt dar, in der Form, dass auch innerhalb der Organisation Hochschule die Systemlogik des Wissenschaftssystems relevant ist, allerdings in eine eigene Organisationslogik übersetzt werden kann und muss. In der Umwelt, also im Wissenschaftssystem, ist das vorherrschende Allokationsprinzip schon immer der Wettbewerb. Der wissenschaftliche Fortschritt wird seit jeher gedacht als Wettbewerb um neue Ideen und Erkenntnisse, bei denen zwar alle Wissenschaftlerinnen und Wissenschaftler ,auf den Schultern von Riesen“" (Merton 1983) stehen, aber auch eine gewisse Konkurrenz untereinander besteht (Schmid/Wilkesmann 2015). Dieser wissenschaftliche Wettbewerb ist aber, wie gezeigt wird, ein inszenierter Wettbewerb im Schatten der Hierarchie. 
Der wissenschaftliche Wettbewerb ist zu differenzieren vom Markt als in unserer Gesellschaft mittlerweile vorherrschender Allokationsmechanismus. ${ }^{1}$ Durch das New Public Management (NPM) findet eine sprachliche Überlagerung und damit Verwechslung beider Allokationsmechanismen statt. Zwar haben beide Verteilungsmechanismen auch gemeinsame Konstitutionseigenschaften: So gibt es weder einen ,natürlichen“ Markt noch einen „natürlichen" Wettbewerb. Beides sind sozial konstruierte Mechanismen. Der Markt muss durch einen dritten Akteur konstitutionell erschaffen und seine Eigentumsordnung durch eben diesen Akteur garantiert werden (Buchanan 1977). Ebenso kann Wettbewerb nur stattfinden, wenn eine Wettbewerbsordnung mit einem Maßstab versehen wird, nach welchen Kriterien der Wettbewerb abläuft. Anschauliche Beispiele für die Schaffung von wissenschaftlichen Wettbewerben sind der Research Excellence Framework (REF), der den Wettbewerb um Drittmittel zwischen den Universitäten regelt (Wilkesmann 2016), sowie das geplante Teaching Excellence Framework (TEF) in England (Land/Gordon 2015), das ein landesweites Ranking nach Lehrqualität erlauben und den oben platzierten Universitäten die Möglichkeit einräumen soll, höhere Studiengebühren als die bisher üblichen $9.000 £$ zu erheben. Hier hat der britische Staat Wettbewerbsregeln eingeführt (REF) oder wird sie in naher Zukunft einführen (TEF), die die Allokation knapper Ressourcen (Forschungsdrittmittel und Studiengebühren) regeln.

Das Marktverhalten dagegen reguliert sich im Unterschied zum wissenschaftlichen Wettbewerb, abstrakt formuliert, nach der Konstitution und der Sicherung der Rahmenordnung über den Preismechanismus selbst. Der dritte Akteur greift (in der Regel) nicht in das Marktgeschehen ein und verfolgt dort keine eigenen Ziele. Anders beim Wettbewerb: Hier definiert der dritte Akteur die Kriterien des Wettbewerbs, gerade um Ressourcen (im wissenschaftlichen Wettbewerb häufig seine eigenen) mit einer bestimmten Zielvorstellung zu verteilen. Im Gegensatz zum Markt greift der dritte Akteur direkt in die Verteilung ein, indem er etwaige Zielzustände vorgibt oder definiert (z.B. die deutsche Exzellenz-Initiative). In diesem Sinne ist der Wettbewerb immer inszeniert im Schatten der Hierarchie, die die Definitionsmacht hat.

Die Einführung des NPM in den letzten 15 bis 20 Jahren im Wissenschaftssystem hat zu einer Überlagerung der wissenschaftlichen Wettbewerbssemantik mit der Marktsemantik geführt. Sie hat mit dazu beigetragen, dass - wie in anderen gesellschaftlichen Subsystemen - die Marktlogik nicht nur dominant, sondern zu einer sich selbst legitimierenden, primären Allokationsfunktion geworden ist. Die sich selbst legitimierende Allokation lässt sich als Fetisch beschreiben, wobei der Begriff Fetisch im Diskurs des Higher Education Research entwickelt wurde, , , [...] to describe an irrational belief

1 Die hier vorgestellte Differenzierung beruht nicht auf dem Koordinationsdreieck nach Clark (1983). 
in magical powers which are invested in an object to protect from harm or to make wishes come true" (Naidoo 2016). Wenn der wissenschaftliche Wettbewerb durch die Überlagerung mit der Marktlogik zum Fetisch wird, besteht die Gefahr, dass dadurch vier wichtige gesellschaftliche Prinzipien ausgehöhlt werden, wie jüngst Hampe (2016) ausgeführt hat: Es besteht das Problem der Zerstörung der vier wichtigen Grundpfeiler menschlichen und wissenschaftlichen Zusammenlebens, nämlich der Empathie, des Vertrauens, der Toleranz und einer übergreifenden Teleologie. Wird die Marktlogik zum selbstlegitimierenden Prinzip, dann geht es für jede Wissenschaftlerin und jeden Wissenschaftler nur darum, den eigenen Preis zu erhöhen, wodurch der für die Wissenschaft so wichtige Perspektivwechsel verschwindet und damit auch die Eigenschaft, mit anderen Wissenschaftlerinnen und Wissenschaftlern zusammen eine reflexive norm- und zielsetzende Gemeinschaft zu begründen (Empathie). Da nur noch jeder sich selbst vertraut, geht wechselseitiges Vertrauen schnell verloren. Es sei denn, es kann - spieltheoretisch gesprochen - ein Vertrauensspiel stabilisiert werden. Dafür ist aber entweder Reputation oder wieder ein dritter Akteur notwendig, der sanktionieren kann (Wiens 2013). Ebenso kann die Anerkennung für fremde (Lebens-)Entwürfe oder neuartige Wege abhanden kommen und durch diese mangelnde Toleranz Innovation übersehen werden oder ganz verloren gehen. Schließlich steht nur noch die eigene Veröffentlichung im A-Journal im Vordergrund. Alles andere zählt nicht mehr. Dabei geht es nur noch um die Anzahl der Artikel in hochrangigen Journalen und deren Impact-Faktor und nicht mehr um den Inhalt derselben oder darum, wie dieser Inhalt generiert wurde. Das Projekt einer langfristigen, gar mehrere Generationen umfassenden Teleologie in der Wissenschaft löst sich auf. Wir stehen zwar noch auf den Schultern von Riesen, aber wir reflektieren dies nicht mehr und geraten damit in die Gefahr, auf den Schultern zu wanken und davon herunterzufallen. Die Leistung von (früheren) Kolleginnen und Kollegen wird ignoriert. Ein gemeinsames Wissenschaftsverständnis oder eine gemeinsame Norm guter wissenschaftlicher Praxis erodiert.

Von Gegnern des NPM im Wissenschaftssystem wird immer Luhmanns Beschreibung des Wissenschaftssystems in der Codierung wahr/unwahr als generalisiertes Kommunikationsmedium fälschlich als unhinterfragbare Legitimation des Status quo verabsolutiert (Luhmann 1990). Aber die Wahrheit ist nie die Leitwährung im Wissenschaftssystem, wie wir spätestens seit Bourdieu (1988) wissen. Die Fakultäten und auch die individuellen Wissenschaftlerinnen und Wissenschaftler sind in einem Kräftefeld eingebunden, in dem die Reproduktion nach Herrschaftskriterien und deren Machtspielen erfolgt. Die Einführung von NPM ist gerade als Versuch zu lesen, diese eingefahrenen Machtspiele zu brechen und neu zu strukturieren. Das reine Rekurrieren auf Wahrheit als ausschließliche Codierung wissenschaftlicher Entscheidung ist damit nach obiger Definition ebenso ein Fetisch wie das Abso- 
lut-Setzen von Marktkriterien. Es stellt ein sich selbst legitimierendes Entscheidungskriterium dar, das aber die eigentliche Allokationsfunktion nur verschleiert.

Auf der Ebene der Organisation, der Hochschulen, wird der Wettbewerbsmechanismus in die Organisationslogik übersetzt, nämlich in eine hierarchische Entscheidung. Die Hierarchie befindet darüber, wer welche Stellen, Ressourcen, Gelder und Aufstiegsmöglichkeiten bekommt. Dazu kann die Organisationsspitze auch einen Wettbewerb via Kennzahlen inszenieren, muss es aber nicht. Die Organisation ist erst in den letzten Jahren über die neuen Steuerungsinstrumente im Rahmen des NPM dazu ermächtigt worden, mit der hierarchischen Entscheidung einen eigenen Allokationsmechanismus einzuführen und sich somit als Organisation von der Umwelt zu emanzipieren. Die knappen Güter, Stellen und Geld, werden in erster Linie durch die Organisation zugewiesen und nicht (mehr) vorrangig durch das Wissenschaftssystem. Dabei hat die Organisation Hochschule nur bedingt Personalund Organisationsmacht (Hüther/Krücken 2012). Personalmacht bezeichnet den Einfluss der Vorgesetzten darauf, ob Mitarbeitende nachgefragte Stellen innerhalb der Organisation bekommen (Luhmann 1975). Früher entschied nicht die Organisation, sondern die Profession über Karrierewege der Wissenschaftlerinnen und Wissenschaftler. Organisationsmacht bezeichnet nach Luhmann (1975) die Möglichkeit, Mitglieder aus der Organisation auszuschließen. Personalmacht hat die Organisation Hochschule im Nachwuchsbereich, aber auch im professoralen Bereich, wenn Tenure-Track-Stellen eingeführt werden, wie z.B. an der TU München oder der Universität DuisburgEssen, die Juniorprofessuren im Regelfall mit tenure ausstattet. Hier kann die Organisation Hochschule sehr wohl auf die Karriere von Wissenschaftlerinnen und Wissenschaftlern Einfluss nehmen. In Nordrhein-Westfalen z.B. beruft die Rektorin oder der Rektor nicht nur die Professorinnen und Professoren, sondern ist auch die Dienstvorgesetzte und den Dienstvorgesetzten. Zwar sind Professorinnen und Professoren als Lebenszeitbeamte nicht kündbar, aber bis zu dieser Auswahl - und bei Tenure-Track-Stellen auch darüber hinaus - bestimmt die Organisation, wer welche knappe Ressource in Form von Stellen bekommt. Sachmittel sowie Mitarbeiterstellen bleiben dauerhafte Steuerungsinstrumente der Organisationsspitze, auch über die Berufung hinaus. Die Organisation Hochschule hat damit gegenüber dem Wissenschaftssystem an Autonomie gewonnen und teilweise Personal- und Organisationsmacht als eigene Entscheidungsoption bekommen bzw. entwickelt. Die Organisation übersetzt das Allokationskriterium der Umwelt in die hierarchischen Entscheidungen: Passt die ausgewählte Person zu den Organisationszielen der jeweiligen Hochschule in Forschung und Lehre? Wie können die Wissenschaftlerin und der Wissenschaftler stärker auf gemeinsame Ziele und Handlungen der Hochschule verpflichtet werden? Die hierarchische Entscheidung 
kann aber auch beinhalten, innerhalb der Organisation einen Wettbewerb zu initiieren und dafür Kennzahlen festzulegen.

Im Folgenden werden einige empirische Evidenzen und Illustrationen aufgeführt, wie diese neuen Steuerungsmechanismen von den Professorinnen und Professoren wahrgenommen werden.

\section{Empirische Evidenzen und Illustrationen: Die Wahrnehmung der Neuen Steuerungsinstrumente}

Datengrundlage der folgenden empirischen Evidenzen sind erstens drei quantitative Befragungen in zwei DFG-Projekten. Die erste Befragung fand zwischen Mai und Juli 2009 unter deutschen Universitätsprofessorinnen und Universitätsprofessoren statt (Wilkesmann/Schmid 2011; Wilkesmann/ Schmid 2012). Es handelt sich dabei um einen repräsentativen Datensatz für alle deutschen Universitätsprofessorinnen und Universitätsprofessoren $(\mathrm{n}=$ 1119). Die zweite Befragung fand im März und April 2011 unter deutschen Fachhochschulprofessorinnen und Fachhochschulprofessoren statt und ist ebenso repräsentativ für die Grundgesamtheit $(\mathrm{n}=942)$ (Wilkesmann 2013). Eine dritte Befragung fand im gleichen Zeitraum statt und erfasste alle Universitäts- und Fachhochschul-Rektorate von Hochschulen in staatlicher Trägerschaft, mit einer Rücklaufquote von 50 Prozent $(\mathrm{n}=47 \mathrm{Uni} ; 53 \mathrm{FH})$ (Schmid/Wilkesmann 2015). Zweitens fließen Auswertungen von neun qualitativen Interviews mit Rektorinnen und Rektoren deutscher Universitäten und Fachhochschulen in die Analyse mit ein. Die Interviews wurden 2016 erhoben, vollständig transkribiert und codiert.

Die hierarchische Steuerung wird durch die Neuen Steuerungsinstrumente (NSI) (Leistungszulagen in der W-Besoldung, Zielvereinbarungen, leistungsorientierte Mittelvergabe) massiv erleichtert und unterstützt. Die Einstellung zu den NSI wirft ein Licht darauf, wie stark die Durchgriffsrechte der Organisation auf die Verteilung knapper Ressourcen innerhalb der Organisation wahrgenommen und wie wichtig diese eingestuft werden. Die NSI ermöglichen und unterstützen die hierarchische Entscheidungsdurchsetzung in der Organisation. Ihre Akzeptanz kann als Proxy für die Akzeptanz hierarchischer Allokation angesehen werden. Zuerst wird die Sichtweise der Rektorinnen und Rektoren dargestellt, anschließend die Wahrnehmung und deren Wandel von allen Professorinnen und Professoren.

Ein erstes interessantes Ergebnis stellt die Differenzierung der Zustimmung nach der Organisationsrolle dar. So stimmen Rektorinnen und Rektoren deutlich stärker den NSI zu als Professorinnen und Professoren (Abb. 1). Dekane und Professorinnen bzw. Professoren unterscheiden sich jedoch kaum. Die Einschätzung der Rektorinnen und Rektoren überrascht auch wenig, da sie mittels dieser Instrumente auf die Allokation knapper Güter in der 
Organisation relativ einfach Einfluss nehmen können und somit Handlungsmacht gewinnen.

Abb.1: Einstellung zu den Neuen Steuerungsinstrumenten nach Organisationsrolle

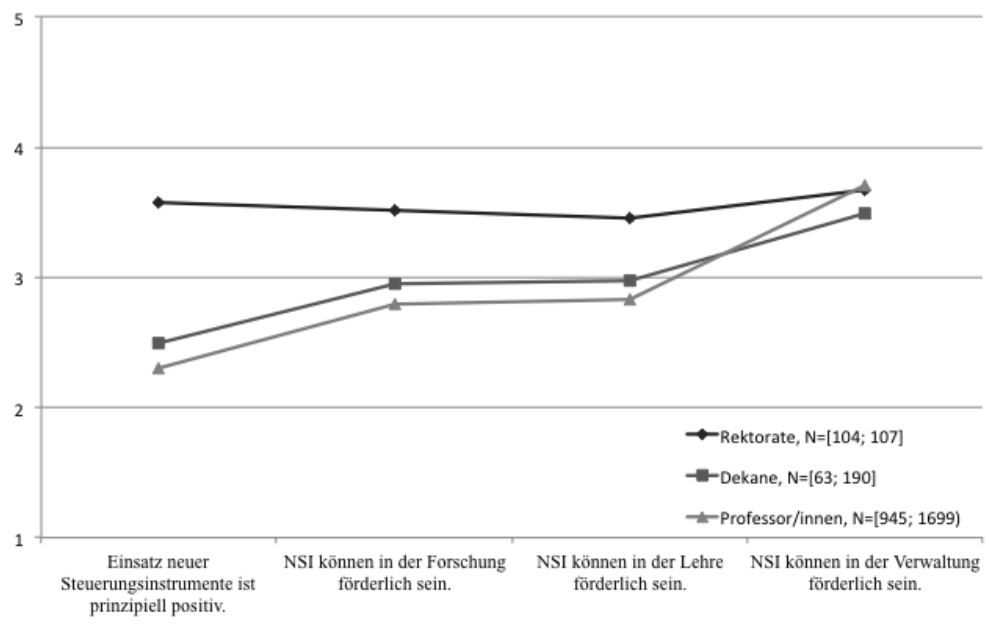

Quelle: eigene Darstellung

Aus den qualitativen Interviews mit Rektorinnen und Rektoren lassen sich drei Steuerungsoptionen entnehmen, die aus deren Sicht besonders wichtig sind: Berufungen, finanzielle Ressourcen und kommunikative Überzeugung. Mit Berufungen wird der Eintritt in die Organisation reguliert und damit die Karriere der Wissenschaftlerinnen und Wissenschaftlern. Finanzielle Ressourcen regulieren die wichtige Ausstattungsfrage nach dem Eintritt. Diese beiden Instrumente sind klassische Optionen der hierarchischen Steuerung und ermöglichen den Rektorinnen und Rektoren erst ihre rollenkonforme Entscheidungsdurchsetzung. Allerdings betonen alle interviewten Rektorinnen und Rektoren, dass beide Steuerungsoptionen immer mit der diskursiven Überzeugung kombiniert werden müssen, um die Professorinnen und Professoren auch in ihrer Selbstbestimmtheit anzuerkennen und ernst zu nehmen. Dies ist eine Frage der guten Führung, dass Entscheidungen erklärt und begründet und nicht einfach ex cathedra verkündet werden, was unter so eigensinnigen Mitgliedern, wie Professorinnen und Professoren, nur Widerstand erzeugen würde.

Aus der Sicht der Professorinnen und Professoren können die Zustimmung und damit die Einschätzung der Bedeutung zu diesen NSI und dem NPM im Allgemeinen in drei idealtypische Gruppen geclustert werden (Schmid/Wilkesmann 2015): 
- Kritikerinnen und Kritiker: Dies sind C-besoldete Naturwissenschaftlerinnen und Naturwissenschaftler, die älter als 54 Jahre sind.

- Indifferente: Hierbei handelt es sich idealtypisch um männliche Gesellschafts- und Sozialwissenschaftlerinnen sowie Gesellschafts- und Sozialwissenschaftler mittleren Alters (46 bis 54 Jahre).

- Befürworterinnen und Befürworter: Hier sind die jungen (unter 46 Jahre), weiblichen Lebenswissenschaftlerinnen zu finden, die in der W-Besoldung sind.

Anhand einer Befragung aus dem Jahre 2001 können Minssen/Wilkesmann (2003) zeigen, dass die Gruppe der Kritikerinnen und Kritiker formelgebundener Mittelzuweisung, die sie „Traditionalisten“-nennen, ca. 75 Prozent aller befragten Professorinnen und Professoren in Nordrhein-Westfalen umfasst. Die Befürworterinnen und Befürworter, „Ökonomisten“ genannt, stellen 25 Prozent der Befragten. Da in der oben genannten Befragung fast identische Items verwendet werden, lässt sich der Befund von 2001 mit den Daten von 2009 vergleichen. Werden nur die Professorinnen und Professoren an Universitäten in NRW betrachtet, welches die Grundgesamtheit in der Untersuchung von Minssen und Wilkesmann (2003) war, so umfasst die Gruppe der Traditionalisten nun mehr nur noch 66 Prozent und die Gruppe der Ökonomisten steigt auf 44 Prozent. Bei aller Vorsicht lässt sich in den acht Jahren zwischen den Befragungen eine Ausweitung der Zustimmung zu den Management-Tools des NPM und damit eine zunehmende Akzeptanz hierarchischer Allokationsinstrumente konstatieren.

Ebenso zeigt ein Vergleich der W-Besoldeten und der C-Besoldeten einen gewissen Sozialisationseffekt. So sind Erstere positiver gegenüber dem NPM eingestellt als Letztere. Desgleichen ist die externale Lehrmotivation der WBesoldeten signifikant höher als die der C-Besoldeten (Wilkesmann/Schmid 2011). Allerdings zeigen auch die beiden Befragungen der Universitäts- und Fachhochschulprofessorinnen und Fachhochschulprofessoren, dass die NSI keinen direkten, unmittelbaren Einfluss auf eine Handlungsebene, nämlich die Lehrhandlungen haben (Wilkesmann/Schmid 2012; Wilkesmann 2013). Nicht eine Leistungsprämie von einhundert Euro ändert die jeweiligen Lehrhandlungen, sondern den größten Einfluss hat die allgemeine Lehrmotivation und andere Sozialisationseffekte, auf die die Organisationsspitze über entsprechende Organisationsstrukturen einen langfristigen, indirekten Einfluss ausüben kann (Wilkesmann/Schmid 2014).

\section{Diskussion: Vermeidung des Fetischs}

Wie kann vermieden werden, dass sowohl auf der Ebene des Wissenschaftssystems wie auf der Ebene der Organisation Hochschule der Wettbewerb zum Markt-Fetisch wird? Die Lösung ist sicherlich am einfachsten auf der Organi- 
sationsebene zu sehen. Hier kann die Hierarchie Entscheidungen über einen kennzahlbasierten Wettbewerb steuern, muss es aber nicht. Wenn die Organisationsspitze Kennzahlen und Indikatoren einführt, dann darf sie diese nicht verabsolutieren, sondern nur als Ausgangspunkt von Verhandlungen und diskursiven Prozessen nehmen. Werden jedoch die Kennzahlen im Sinne eines MarktFetischs als selbstlegitimierend absolut gesetzt, dann wird der im akademischen Kontext sehr wichtige Aspekt der Selbstbestimmung und der Selbstverpflichtung verletzt. Daten und Indikatoren des Wettbewerbs sollen Anlass und Ausgangspunkt von Kommunikation sein, in der gemeinsam Ziele definiert werden. Dieser Ausgangspunkt ist als Kommunikationsanlass von großer Bedeutung, weil sonst nicht klar ist, über was genau geredet wird. Sie sind aber auch kein Selbstzweck, der nur exekutiert wird (Wilkesmann 2015). Ähnliche Überlegungen gelten für die Ebene des Wissenschaftssystems. Auch hier sollten die Kennzahlen des Wettbewerbs nur Grundlage einer politisch-diskursiv begründeten Entscheidung sein und nicht verabsolutiert werden.

Uwe Wilkesmann, Prof. Dr., ist Direktor des Zentrums für HochschulBildung an der Technischen Universität Dortmund.

\section{Literatur}

Bourdieu, Pierre (1988): Homo Academicus. Oxford: Polity Press.

Buchanan, James M. (1977): Freedom in Constitutional Contract. Texas: A\&M University Press.

Clark, Burton R. (1983): The Higher Education System: Academic Organization in Cross-national Perspective. Berkeley u.a.: University of California Press.

Hampe, Michael (2016): Warum lügen und betrügen Wissenschaftler. In: Die Zeit, Nr. 20 vom 4. Mai 2016.

Hüther, Otto/Krücken, Georg (2012): Hierarchie ohne Macht? Karriere- und Beschäftigungsbedingungen als ,vergessene“ Grenzen der organisatorischen Umgestaltung der deutschen Universitäten. In: Wilkesmann, U./Schmid, C. J. (Hrsg.): Hochschule als Organisation. Wiesbaden: Springer VS, S. 27-39.

Land, Ray/Gordon, George (2015): Teaching Excellence Initiatives: Modalities and Operational Factors. York: The Higher Education Academy.

Luhmann, Niklas (1975): Macht. Stuttgart: Enke.

Luhmann, Niklas (1990): Die Wissenschaft der Gesellschaft. Frankfurt am Main: Suhrkamp.

Merton, Robert K. (1983): Auf den Schultern von Riesen. Frankfurt am Main: Suhrkamp.

Minssen, Heiner/Wilkesmann, Uwe (2003): Lassen Hochschulen sich steuern? In: Soziale Welt 54, 2, S. 123-144. 
Naidoo, Rajani (2016): The competition fetish in higher education: varieties, animators and consequences. In: British Journal of Sociology of Education 37,1, S. 1-10.

Schmid, Christian J./Wilkesmann, Uwe (2015): Ansichtssache Managerialismus an deutschen Hochschulen - Ein empirisches Stimmungsbild und Erklärungen. In: Beiträge zur Hochschulforschung 37, 2, S. 56-87.

Wiens, Marcus (2013): Vertrauen in der ökonomischen Theorie. Eine mikrofundierte und verhaltensbezogene Analyse. Münster: LIT-Verlag.

Wilkesmann, Uwe (2013): Effects of transactional and transformational governance on academic teaching - empirical evidence from two types of higher education institutions. In: Tertiary Education and Management 19, 4, S. 281-300.

Wilkesmann, Uwe (2015): Imaginary contradictions of university governance. In: Welpe, I. M./Wollersheim, J./Ringelhan, S./Osterloh, M. (Hrsg.): Incentives and Performance - Governance of Research Organizations. Cham: Springer, S. 189-205.

Wilkesmann, Uwe (2016): Teaching matters, too - Different ways of governing a disregarded institution. In: Leisyte, L./Wilkesmann, U. (Hrsg.): Organizing Academic Work in Higher Education. Teaching, Learning, and Identities. New York: Routledge, S. 33-54.

Wilkesmann, Uwe/Schmid, Christian J. (2011): Lehren lohnt sich (nicht)? Ergebnisse einer deutschlandweiten Erhebung zu den Auswirkungen leistungsorientierter Steuerung auf die universitäre Lehrtätigkeit. In: Soziale Welt 62, 3, S. 251-278.

Wilkesmann, Uwe/Schmid, Christian J. (2012): The impacts of new governance on teaching at German universities. Findings from a national survey. In: Higher Education 63, 1, S. 33-52.

Wilkesmann, Uwe/Schmid, Christian J. (2014): Intrinsic and internalized modes of teaching motivation. In: Evidence-based HRM 2, 1, S. 6-27. 